\title{
El pago por servicios ambientales en fincas ganaderas: una percepción de productores de Matiguás, Nicaragua
}

\author{
Claudia J. Sepúlveda ${ }^{1}$, Yuri Marín², Muhammad Ibrahim³ y Elías Ramírez ${ }^{4}$ \\ Grupo Ganadería y Medio Ambiente (GAMMA) del Centro Agronómico Tropical de Investigación y Enseñanza (CATIE), CATIE 7170, \\ Turrialba, Costa Rica.E-mail: csepul@catie.ac.cr \\ Instituto de Investigación y Desarrollo Nitlapan de la Universidad Centroamericana (UCA), Apdo. 69 Managua, Nicaragua \\ Grupo Ganadería y Medio Ambiente (GAMMA) del Centro Agronómico Tropical de Investigación y Enseñanza (CATIE), CATIE 7170, \\ Turrialba, Costa Rica. \\ Instituto de Investigación y Desarrollo Nitlapan de la Universidad Centroamericana (UCA), Apdo. 69 Managua, Nicaragua
}

Recibido: julio de 2007 / Aceptado: julio de 2007

EL CATIE Y NITLAPAN ESTÁN IMPLEMENTANDO UN PROYECTO EN Nicaragua para evaluar el impacto que tiene el pago por servicios ambientales (PSA) en la adopción de sistemas silvopastoriles (SSP). El pago fue calculado mediante un índice de uso del suelo desarrollado por el proyecto y los finqueros lo han estado recibiendo durante los últimos 4 años. Se han desarrollado estudios para determinar el efecto del PSA sobre los cambios de uso del suelo y sus implicaciones socioeconómicas y productivas en diferentes niveles de pobreza, y también para consultar las percepciones que ellos tienen sobre el esquema implementado por el proyecto. En este estudio reportamos la percepción que tienen los finqueros de diferentes estratos de pobreza ('pobres' y 'no-pobres') acerca del PSA, su operatoria y el impacto sobre la reducción de la pobreza. Los resultados indican que los finqueros de diferentes estratos de pobreza tienen interés en continuar produciendo bajo esquemas de PSA. Sin embargo, indican que tiene fuertes limitaciones principalmente financieras y por lo tanto es necesario que se diseñen políticas que provean incentivos como créditos verdes para la inversión en los cambios de uso de la tierra. Se concluye también que el PSA es una importante herramienta para promover usos sostenibles de la tierra en fincas ganaderas sin importar el estrato de pobreza. Asimismo, que los finqueros clasificados como 'pobres', pueden beneficiarse de los sistemas de PSA porque contribuyen al incremento de los indicadores socioeconómicos de sus fincas, y por ende, a un mayor bienestar familiar.

Palabras clave: recursos naturales-valoración / servicios ambientales / granjasMatiguás, Matagalpa (Nicaragua) / agricultura-aspectos económicos / uso de la tierra 


\section{Introducción}

La ganadería es una actividad a la que se dedica más de la mitad de las familias rurales en América Central. Las tierras para esta actividad ocupan alrededor del $60 \%$ de la superficie en las fincas (Casasola et al., 2006). Sin embargo, aproximadamente un $40 \%$ de las pasturas en América Central está degradada (Szott et al., 2000), lo que está asociado a una baja productividad y rentabilidad en las fincas ganaderas (Betancourt et al., 2006; Holmann \& Estrada, 1997). Por otra parte, un promedio porcentual de los pobres rurales vive en áreas de bajo potencial productivo, incluyendo zonas degradadas donde la ganadería es predominante (Espinoza et al., 1999). Para revertir los procesos de degradación de pasturas y mejorar las condiciones agroecológicas y socioeconómicas de las fincas, se está fomentando la replicación de sistemas silvopastoriles en fincas ganaderas debido a las bondades que estos sistemas tienen para mejorar la productividad de las fincas y la generación de servicios ambientales (Chagoya, 2004). En América Central, para los productores ganaderos más pobres, la falta de capital para el establecimiento de estas tecnologías representa una barrera de adopción y replicación (López, 2005). En ese sentido, los pagos por servicios ambientales (PSA) representan una buena alternativa para incentivar la adopción de los sistemas silvopastoriles en fincas con predominio de uso ganadero (Pagiola et al., 2004).

Últimamente existe mucho interés entre donantes y diseñadores de políticas para que el PSA sea un instrumento que contribuya a mejorar el bienestar de las familias rurales, pues se argumenta que éste, además de posibilitar la conservación de los recursos naturales, bajo determinadas circunstancias puede contribuir a mejorar las condiciones de vida de los productores más pobres al introducir en sus fincas prácticas más sostenibles que les permitan aumentar la productividad y generar ingresos adicionales a través de la venta de servicios ambientales ( Ibrahim et al., 2005; Pagiola et al.,2005).

En los últimos cinco años, el Centro Agronómico Tropical de Investigación y Enseñanza (CATIE) en conjunto con el la Fundación Centro para la Investigación en Sistemas Sostenibles de Producción Agropecuaria (CIPAV) en Colombia y el Instituto de Investigación y Desarrollo (Nitlapan) de la Universidad Centroamericana (UCA), con financiamiento del GEF, LEADFAO y el Banco Mundial, han implementado el proyecto Enfoques Silvopastoriles Integrados para el Manejo de Ecosistemas con el propósito de evaluar si el PSA constituye una herramienta que fomenta la implementación de usos de la tierra más sostenibles en las fincas ganaderas. Los resultados preliminares muestran que el PSA ha provocado cambios importantes hacia los sistemas silvopastoriles a cuenta de reducir las pasturas degradadas (Zapata et al., 2006). La replicación de los sistemas silvopastoriles y el éxito de los PSA dependen del grado de satisfacción del mecanismo de pago que tengan los productores y sus familias y de si están satisfechos con el valor del incentivo que reciben para realizar los cambios de uso de la tierra en sus fincas.

En este sentido, el presente artículo analiza la opinión de los productores según su condición de pobreza - 'pobres' y 'no-pobres' - en la zona piloto del proyecto GEF/silvopastoril en Matiguás, Nicaragua en relación a cómo perciben el PSA y cómo toman sus decisiones de cambio en usos del suelo, así como su percepción sobre las barreras de adopción que enfrentan para hacer los cambios, sus preferencias por determinados usos, su percepción sobre la importancia de la asistencia técnica y sus perspectivas de cambio a futuro en una 
situación sin proyecto. En síntesis, se analiza bajo qué condiciones los PSA pueden llegar a ser un instrumento efectivo para favorecer cambios sostenibles en el uso del suelo en fincas ganaderas, en particular en los más 'pobres', y qué medidas de políticas podrían pensarse para replicar estos cambios.

\section{Materiales y métodos}

\section{1. Área de estudio}

Este estudio se realizó en Matiguás, ubicado en el Centro Geográfico de Nicaragua en el departamento de Matagalpa, aproximadamente a $140 \mathrm{~km}$ de Managua, con una elevación que varía entre 200 y 400 msnm. La precipitación del área fluctúa entre 1200 y 1800 mm anuales, distribuido entre los meses de mayo a diciembre (Ruiz, 2002).

El área de estudio se localiza en dos microcuencas ganaderas, Bul Bul y Paiwas, ambas zonas de intervención del Proyecto Enfoques Silvopastoriles Integrados para el Manejo de Ecosistemas. El uso de la tierra de ambas microcuencas está dominado por pasturas, representando aproximadamente el 60\% del área total. De éstas, aproximadamente la mitad estaba degradada y con baja cobertura arbórea. Los cultivos anuales representan una pequeña parte de la superficie total y el bosque (principalmente ripario) representa aproximadamente el 20\% del área total. El tamaño de las fincas oscila entre 10 hasta 70 ha. Los productores se dedican casi en forma exclusiva a la ganadería de doble propósito. La mayoría es relativamente pobre ${ }^{1}$ sus fincas carecen de servicios básicos como energía eléctrica y agua potable (Levard et $a l ., 2000)$.

\subsection{Selección de la muestra}

En 2003 se implementó el proyecto arriba mencionado con el objetivo de evaluar el impacto del PSA sobre la adopción de sistemas silvopastoriles, y otros usos del suelo amigables con el ambiente, para generar servicios ambientales globales y mejorar las condiciones socioeconómicas de las fincas ganaderas. Para tal efecto, se seleccionó un grupo de 131 productores entre pequeños y medianos. Este grupo grande de productores se dividió en 3 grupos de atención: a) los que recibieron sólo PSA $(n=29)$, b) los que recibieron PSA y asistencia técnica (AT; $n=75)$ y c) un Grupo de Control $(n=27)$.

Para efectos de este estudio se seleccionó una muestra de productores $(n=87)$ de todos los que recibieron pago durante el período 2003-2006: a) los que recibieron sólo PSA y b) los que recibieron PSA y asistencia técnica, a quienes se les aplicó una encuesta estructurada para colectar información cualitativa. El total de la muestra fue de 87 encuestados $(n=87)$.

\subsection{Clasificación de los estratos de pobreza}

Los productores seleccionados se clasificaron en dos estratos de pobreza ('pobres' y nopobres) utilizando la clasificación de pobreza elaborada por Marín et al., (2006) en base a la metodología utilizada por Munk (2004), que está basada en un conjunto de indicadores sociales y económicos adaptados a la zona y que contienen las siguientes variables: a) 
activos (tenencia de tierra, ganado, equipos e infraestructura); b) nivel de escolaridad; c) condiciones de vida (calidad de la vivienda y acceso a servicios de agua y electricidad); d) diversificación de las fuentes de ingresos; y e) condiciones de acceso al mercado. Cada indicador está basado en un sistema de puntos (escala) que evidencia diferentes grados de pobreza y con estos indicadores se construyó un índice de pobreza que varía entre 0 (menor pobreza) y 1 (mayor pobreza). El valor del índice para cada productor resulta del promedio ponderado de puntos obtenidos en cada uno de los indicadores. Con esta información se establecieron tres categorías de 'pobres' rurales: 1) 'no-pobres'; 2) 'menos pobres'; y 3) 'más pobres'. Para fines del presente estudio, los productores 'menos' y 'más pobres' se agruparon en un solo estrato, quedando finalmente dos categorías: 1) 'pobres' (n=53) y 'nopobres' $(\mathrm{n}=34)$.

\subsection{Procesamiento de la información}

Para obtener la percepción de los productores, el principal instrumento utilizado para capturar la información fue una encuesta semi-estructurada que en cuanto a tiempo, costo y tamaño de muestra resultó ser el instrumento más eficiente. De manera complementaria se utilizaron otros métodos de acercamiento a la realidad durante las visitas de campo, como la observación participante, las entrevistas informales y sondeos. Las áreas de percepción exploradas fueron: a) el esquema de PSA implementado, su confianza, limitaciones y su visión de las instituciones que lo implementan; b) las motivaciones para hacer los cambios de uso de la tierra; c) la valoración de los diferentes usos del suelo en términos productivos y ambientales; d) la importancia y calidad de la asistencia técnica y su relación con la generación de los servicios ambientales; e) las perspectivas de los productores sobre sus planes a futuro; f) las preferencias sobre esquemas de PSA más apropiados; g) el pago de la certificación; y h) las medidas de política o incentivos para la replicación de los cambios.

Para homogenizar el uso de las preguntas y respuestas de la encuesta por parte de los encuestadores, se realizó un taller instructivo para poner a prueba el manejo de las claves y las formas de abordaje del entrevistado. Mediante una encuesta piloto y la lectura de la realidad en cada zona se resolvieron algunos problemas relacionados con su aplicación y confiabilidad. Mediante el ordenamiento de los valores correspondientes a las respuestas se obtuvieron las frecuencias absolutas y posteriormente las frecuencias relativas. Se consideró una respuesta predominante cuando la frecuencia relativa superó el 50\%. Para el procesamiento de datos se utilizó el Programa Infostat.

Para entender un poco más la realidad de los productores, la discusión y las conclusiones se realizaron desde la opinión (percepción) de éstos, y las tendencias generales fueron relacionadas con variables que explican los impactos del proyecto, las lecciones aprendidas y las limitaciones encontradas a lo largo de cuatro años y medio de implementación. 


\section{Resultados y discusión}

\subsection{Sobre las razones del pago y las motivaciones para realizar los cambios}

En términos generales, un alto porcentaje respondió que el pago que recibió del proyecto es por la venta de los servicios ambientales que genera en sus fincas, el mayor porcentaje (67.3\%) corresponde al grupo de productores 'pobres' comparado con el de 'no-pobres' (56.7\%) (Cuadro 1). Es grato observar que los productores realizan sus cambios de uso del suelo motivados por la importancia de la conservación de los recursos naturales y convencidos de que una manera más racional de producir los conducirá a la generación de un servicio ambiental que posteriormente puede ser valorado económicamente, y consecuentemente traer más beneficios económicos y sociales a ellos y a sus familias. Esto es importante tomando en cuenta que hay un fuerte interés de parte de los donantes en que se implementen más programas de PSA que permitan contribuir con el bienestar y mejoramiento de las condiciones de vida, especialmente de los pequeños y medianos productores.

Por otro lado, más del $28.7 \%$ de los productores 'no-pobres' responden que el pago fue por incrementar la producción ganadera. Estos grupos de productores generalmente están más orientados hacia los mercados y por eso siempre tienen mucho interés de incrementar la productividad en sus fincas (López, 2005).

Cuadro 1. Percepción sobre las razones del pago que realiza el Proyecto Silvopastoril (porcenta espuestas)

\begin{tabular}{|l|c|c|}
\hline \multicolumn{1}{|c|}{ Razones } & $\begin{array}{c}\text { 'pobres' } \\
\mathrm{n}=53\end{array}$ & $\begin{array}{c}\text { 'no - pobres' } \\
\mathrm{n}=34\end{array}$ \\
\hline Venta de servicios ambientales & 67.3 & 56.7 \\
\hline Incrementar la producción ganadera & 19.8 & 28.7 \\
\hline Subsidio & 4.0 & 5.6 \\
\hline Otros & 8.9 & 9.0 \\
\hline
\end{tabular}

Por otra parte, a los productores se les preguntó sobre las motivaciones que los llevaron a realizar los cambios de uso del suelo en sus fincas. Las motivaciones que llevan a los productores 'pobres' y 'no-pobres' a realizar los cambios son prácticamente las mismas y se dan por diferentes razones. Más del 24\% de los productores 'pobres' y 'no-pobres' se sienten motivados a realizar los cambios de uso por una razón técnica, el aumento de la productividad en su sistema de producción ganadera.

Estos dos grupos también consideran las razones de tipo ambiental como importantes. Por ejemplo, el 16.6\% de los productores 'pobres' y el 20.7\% de los 'no-pobres' ven el PSA como una oportunidad para conservar el medio ambiente. Esto indica que los productores tienen un gran interés en producir conservando los recursos naturales y demuestra que existe, por parte de los finqueros, una mayor conciencia y valoración de las prácticas que implementan con éxito, lo que corresponde al esfuerzo económico y al interés de innovar en sistemas de producción más sostenibles para la generación de servicios ambientales. 
No obstante, nuevamente el factor de pago se presenta como una razón de peso para que el productor se motive con un programa de PSA. Por ejemplo el 18.6\% de los productores 'pobres' y el 16.1\% de los 'no-pobres' respondieron que el otorgamiento del pago los motiva a hacer cambios de uso del suelo porque consideran que el monto que reciben alivia o solventa situaciones específicas de falta de liquidez en sus familias. Esto es muy significativo si se toma en cuenta que la mayoría de los productores tiene como limitante la falta de capital para hacer cambios en sus fincas. Como complemento a este análisis, más adelante se detallan datos del destino final que los productores le dan a los montos por PSA.

Finalmente, existe otro grupo de productores - 19.3\% de los 'pobres' y 17.2\% de los 'nopobres' - que tiene razones conservadoras y que prefiere mantener las condiciones naturales actuales de su finca. No implementan nuevas tecnologías pero mantienen las que ya han implementado, especialmente en lo que se refiere a las pasturas mejoradas. En el Cuadro 2 se muestran otras razones que motivan a los productores a hacer cambios.

Cuadro 2. Razones que motivan a los productores a hacer cambios de uso del suelo (Porcentaje de respuestas)

\begin{tabular}{l|cc}
\hline Motivos & $\begin{array}{c}\text { 'pobres' } \\
(\mathrm{n}=53)\end{array}$ & $\begin{array}{c}\text { 'no-pobres' } \\
(\mathrm{n}=34)\end{array}$ \\
\hline El otorgamiento del pago & 18.6 & 16.1 \\
Aumento de la producción ganadera & 24.1 & 25.3 \\
Mejoramiento de las condiciones naturales & 19.3 & 17.2 \\
Aumento del valor agregado & 11.7 & 17.2 \\
Mejoramiento de la rentabilidad & 9.7 & 3.4 \\
Mejoramiento de las condiciones ambientales & 16.6 & 20.7 \\
\hline
\end{tabular}

\subsection{La inversión de los productores del monto de PSA recibido del proyecto}

Si bien se sabe que los programas de PSA en sistemas ganaderos se diseñan como un medio para detener o evitar la degradación de tierras dedicadas a esta actividad, también se puede entender que el PSA es un mecanismo que contribuye a aliviar medianamente los niveles de pobreza en los medios rurales. Este planteamiento tiene se fundamenta observando las respuestas que hacen los finqueros a la pregunta: ¿en qué invierte mayoritariamente el monto de PSA que recibe del proyecto? El 49.2\% de los productores clasificados como 'pobres' y el $53.8 \%$ de los clasificados como 'no-pobres' responden que utilizan el dinero mayoritariamente para realizar cambios de uso del suelo. Más del 30\% de ambos grupos anota que el dinero lo destina para realizar diferentes inversiones en la finca, como construcción y mantenimiento de infraestructura, compra o reparación de equipos. Esto significa que más del 75\% de los montos del pago, en ambos grupos, ha sido dirigido a capitalizar las fincas. Sin embargo, como se observa en el Cuadro 3, los 'pobres' invierten relativamente un poco menos que los ‘no-pobres' porque destinan una mayor proporción del monto recibido a pagar deudas y cubrir otras necesidades. 
Cuadro 3. Inversión del monto de PSA recibido por los productores (porcentaje de respuestas)

\begin{tabular}{|l|c|c|}
\hline \multicolumn{1}{|c|}{ Inversión } & $\begin{array}{c}\text { 'pobres' } \\
\mathrm{n}=53\end{array}$ & $\begin{array}{c}\text { 'no -pobres' } \\
\mathrm{n}=34\end{array}$ \\
\hline Cambios de uso del suelo & 49.2 & 53.8 \\
\hline Inversiones en la finca (Infraestructura, equipos, etc.) & 30.6 & 35.4 \\
\hline Mejora de las condiciones de la familia & 3.2 & 4.0 \\
\hline Pago de deudas & 7.8 & 3.8 \\
\hline Ahorro & 0.4 & 0.0 \\
\hline Compra de animales & 1.4 & 0.6 \\
\hline Otras & 7.5 & 2.4 \\
\hline
\end{tabular}

\subsection{Sobre el esquema de PSA en el que participan}

Es importante resaltar que los productores tienen confianza no sólo en el esquema de PSA propuesto por el proyecto, sino también en las instituciones que lo promueven. En el caso de Nicaragua, es NITLAPAN - UCA la institución que realiza los trámites necesarios para que los productores ingresen al esquema y se encarga también de realizar directamente los pagos. Los productores son generosos en sus apreciaciones. Más del 94\% de los productores de ambos grupos de pobreza manifiestan tener confianza en ella y sólo cerca del $6 \%$ de los productores 'no-pobres' expresa que no tienen confianza en la institución. Como se observa en la Ilustración 1, la no confianza tiene que ver con las respuestas de los productores que no han recibido acompañamiento de la asistencia técnica (grupo de sólo pago) y que no entendieron suficientemente bien el funcionamiento del esquema, situación que es normal, ya que no siempre todos los implicados en un proceso innovador podrán mostrar satisfacción total.

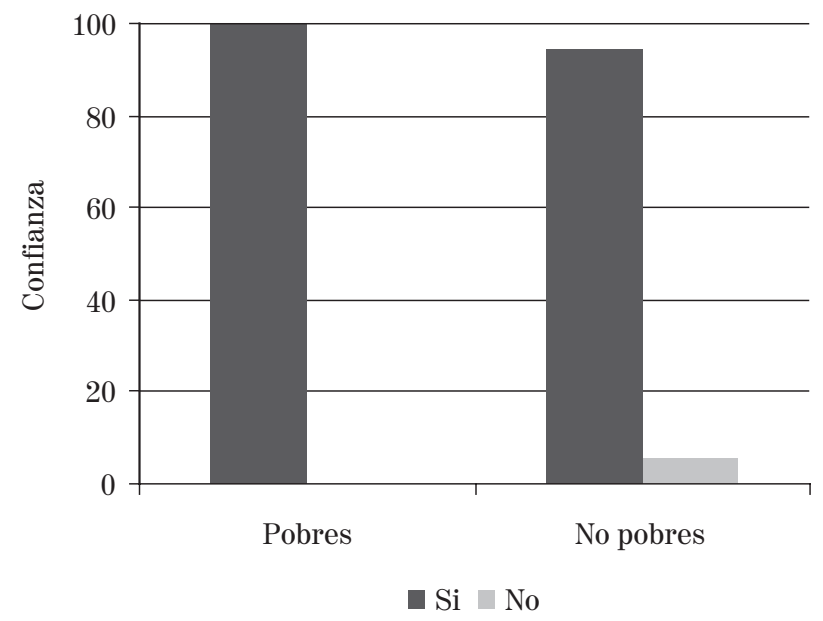

Ilustración 1. Confianza en el esquema de PSA y las instituciones que lo proponen (porcentaje de respuestas) 


\subsection{Los montos del PSA}

Tal vez el aspecto más sensible del programa de PSA es la cantidad que recibe cada productor por realizar cambios de uso del suelo y generar servicios ambientales en su finca. A los productores se les preguntó si estaban satisfechos con el monto de pago recibido por año (o que recibió en el caso del esquema de dos años). Como se observa en la Ilustración 2, hay respuestas cercanas entre el grupo de productores 'pobres' y 'no-pobres'. La mayoría, el 57\% de los primeros y el 64\% de los segundos, respondió haber recibido un monto de dinero de acuerdo con lo que esperaban. Un 32\% de los productores 'pobres' y el 17\% de los 'no-pobres' dijo haber recibido menos de lo que esperaba, mientras un poco más del 10\% de ambos grupos dijo recibir un monto mayor al esperado. Eso significa que el pago, además de contribuir para que las familias tengan un nivel de ingresos superior al normal, también permitió que mediante el factor sorpresa, al no esperar un monto adicional de dinero, los finqueros se motivaran más a realizar cambios en sus fincas y a continuar produciendo bajo esquemas de PSA.

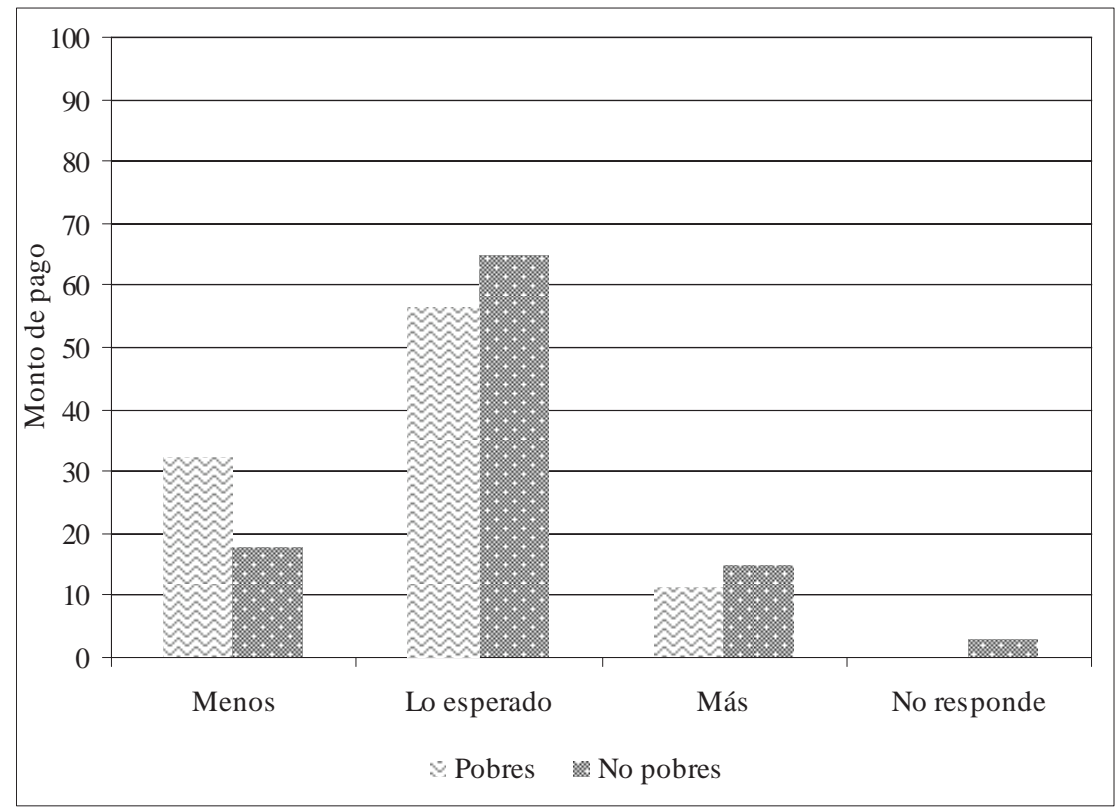

Ilustración 2. Calificación del monto del pago recibido por los productores 'pobres' y ‘nopobres' (porcentaje de respuestas)

\subsection{Importancia de la asistencia técnica en los cambios de uso del suelo}

Ya conocemos que el PSA tiene un efecto positivo en la implementación de los sistemas silvopastoriles. Sin embargo, pese a sus bondades es indispensable el acompañamiento por medio de la capacitación y asistencia técnica para lograr cierto tipo de promoción de los sistemas, especialmente por parte de los técnicos de las instituciones que promueven estos esquemas de PSA. Esto lo indica cierta tendencia mayor a cambiar en las fincas que 
reciben asistencia técnica con respecto a las que no la reciben, en magnitud y calidad del establecimiento y manejo de los cambios. A futuro, esto se podría notar con una mayor persistencia y respuesta productiva y/o de conservación de los diferentes usos del suelo implementados en las fincas.

Los productores consideran que la asistencia técnica es un elemento muy importante para la sostenibilidad del programa PSA, más del 95\% de las respuestas así lo indican (ver los resultados en el Cuadro 4).

Cuadro 4. Importancia de la asistencia técnica (porcentaje de respuestas)

\begin{tabular}{|l|c|c|}
\hline \multicolumn{1}{|c|}{ Importancia } & $\begin{array}{c}\text { 'pobres' } \\
\mathrm{n}=53\end{array}$ & $\begin{array}{c}\text { 'no-pobres' } \\
\mathrm{n}=34\end{array}$ \\
\hline Muy importante & 97.4 & 95.8 \\
\hline Poco importante & 2.6 & 4.2 \\
\hline No es importante & 0.0 & 0.0 \\
\hline
\end{tabular}

Más del 67.7\% de los productores de ambos grupos desea tener el acompañamiento de la asistencia técnica de manera permanente, mientras que un 32\% de los productores opinó que desea un acompañamiento de mediano plazo, 3 años sería suficiente. En la Ilustración 3 se puede observar el porcentaje de respuesta. También se les consulto si estarían dispuestos a invertir un porcentaje del monto que reciben por PSA para pagar servicios de asistencia técnica, a lo que un 59.4\%, respondió afirmativamente.

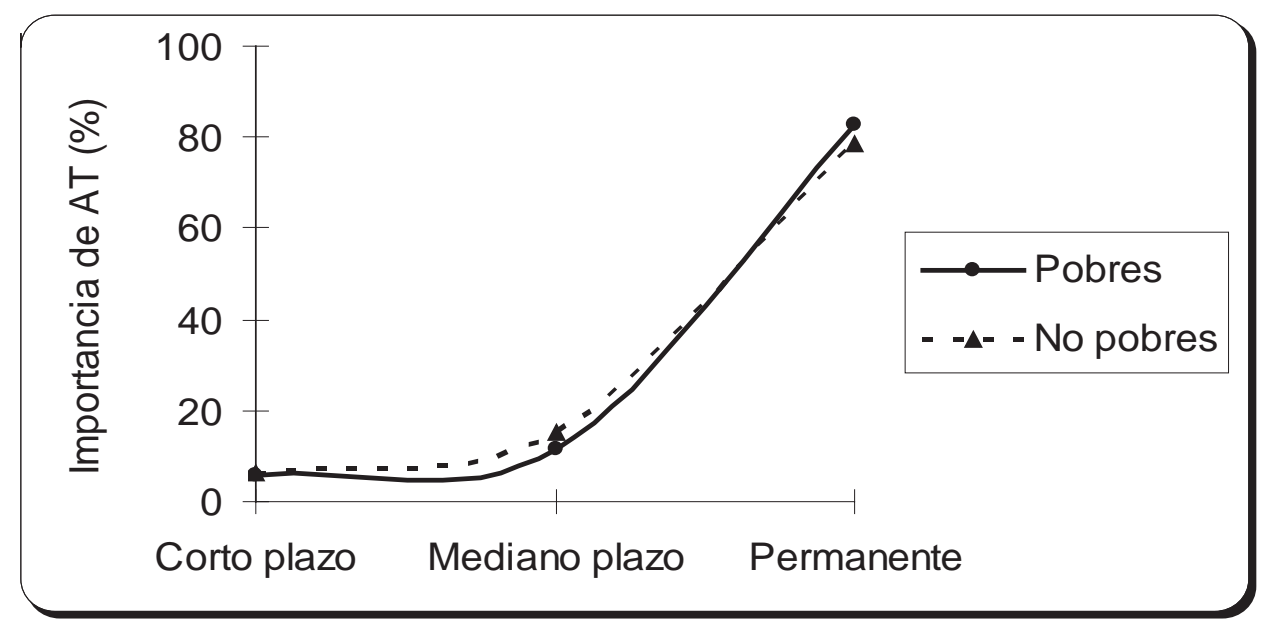

Ilustración 3. Período de acompañamiento al productor (porcentaje de respuestas)

\subsection{Limitantes y barreras del PSA}

Las principales limitantes que tienen los productores para participar en un programa de PSA se presentan en el Cuadro 5. La disponibilidad de la tierra y el acceso a recursos financieros 
para realizar las inversiones que les permitan acceder al pago, fueron los dos factores más importantes que limitan su participación en los esquemas de PSA.

Cuadro 5. Limitantes de la finca y la familia para recibir un mayor pago los servicios ambientales. (Porcentaje de respuestas)

\begin{tabular}{lcc}
\hline \multicolumn{1}{c}{ Limitantes } & $\begin{array}{c}\text { 'pobres' } \\
\mathrm{n}=34\end{array}$ & $\begin{array}{c}\text { 'no - pobres' } \\
\mathrm{n}=53\end{array}$ \\
\hline Disponibilidad de la tierra & 28.4 & 25.7 \\
Acceso al capital y/o financiamiento & 39.7 & 31.4 \\
Disponibilidad de mano de obra contratada & 12.1 & 20.0 \\
Capacitación y asistencia técnica & 6.0 & 8.6 \\
Condiciones climáticas & 12.9 & 14.3 \\
\hline
\end{tabular}

El sistema de PSA implementado por el proyecto dio un mayor valor a los usos de la tierra con tacotales, plantaciones forestales y bosques. Esto significa que si los productores quieren optar por este tipo de usos, deberían de liberar algunas áreas de sus fincas que están bajo pastoreo y por esa razón ellos identificaron la disponibilidad de la tierra como una limitante para realizar más cambios de uso de la tierra.

Como se esperaba, los finqueros más 'pobres' mencionaron en mayor proporción (39.7\%) la falta de financiamiento como su principal limitante para hacer los cambios. Los resultados del Proyecto Silvopastoril muestran que los sistemas de uso de la tierra, como potreros mejorados con alta densidad de árboles, necesitan una inversión de 160 dólares por ha y los bancos forrajeros para su establecimiento hasta 600 dólares por ha (Casasola et al., 2006). En relación con estos costos, muchos productores 'pobres' no tienen los recursos ni el financiamiento necesario para invertir en estos sistemas. Para solventar este problema es importante que el proyecto modifique su metodología de PSA y genere herramientas para que los diseñadores de políticas institucionalicen políticas, como los créditos blandos, que estén disponibles para los productores de manera que éstos puedan invertir en las diferentes tecnologías propuestas. Generaría un mayor éxito para los proyectos si todos los estratos de productores pudieran participar en un programa de PSA. Además, también sería interesante que posteriormente se puedan considerar estrategias para reducir costos de transacción que son altos para los productores pequeños vs. grandes según lo indican datos del Proyecto 2007.

Por otro lado, el 20\% de los productores 'no-pobres' indica que la disponibilidad de mano de obra es una limitante y esto se puede relacionar directamente con la dependencia que tienen las fincas más grandes de mano de obra contratada. En cambio, solamente un 12\% de los productores 'pobres' mencionó que la disponibilidad de mano de obra sea una limitante importante para hacer cambios en el uso de la tierra y recibir un mayor pago. Los datos del proyecto y otros resultados de esta encuesta muestran que, por lo general, estas familias 
tienen un número promedio de seis miembros, por lo que tienen menos dependencia de mano de obra contratada para realizar cambios. Los dos grupos de productores no identifican la capacitación y la asistencia técnica como un factor muy limitante para los cambios. Esto posiblemente esté relacionado a la relativa buena oferta de asistencia técnica que existe en la zona.

\subsection{Valoración productiva y ambiental de los diferentes usos del suelo}

Alos productores se les solicitó su valoración sobre cuáles usos de la tierra consideraban más importantes tanto para la producción como para la conservación. En términos productivos, ambos grupos de productores se mostraron de acuerdo en que los bancos forrajeros y las pasturas mejoradas con árboles son los usos del suelo considerados más importantes para la producción ganadera en su finca (Ilustración 4). Asimismo, ambos grupos de productores (más del 14\%) consideran los bosques riparios y los tacotales como los usos de tierra menos importantes en términos productivos.

Recientemente muchos productores en la zona de Matiguás están sembrando bancos forrajeros como una estrategia de alimentación de los animales en la época seca. Esta táctica es de mucha importancia debido a que evita que se baje considerablemente la producción ganadera en esta época y contribuye a disminuir el sobrepastoreo, reduciéndose la erosión de los suelos cuando vuelven las primera lluvias (Szott et al., 2000).

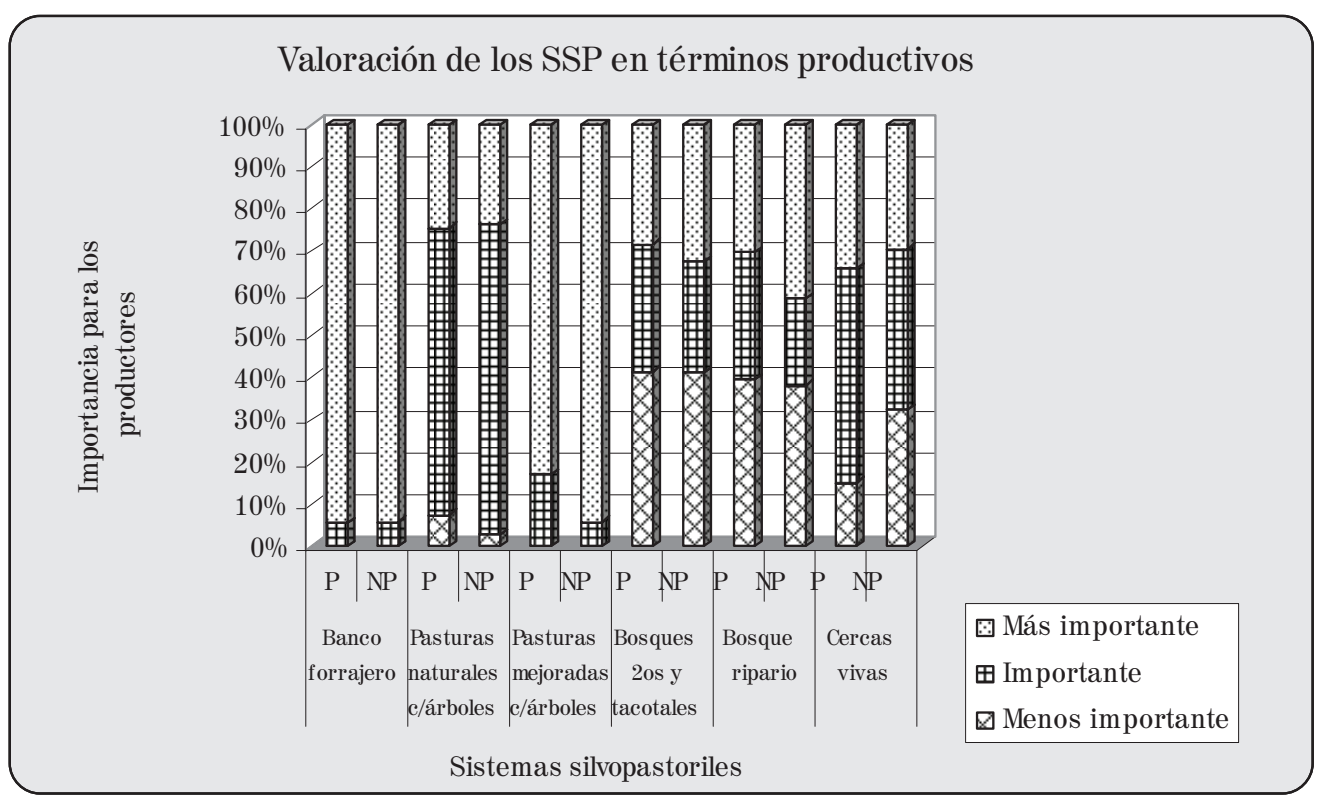

Ilustración 4. Valoración productiva de los usos del suelo por parte de los productores (Porcentaje de respuestas)

En cuanto a la conservación, los productores mencionaron que los bosques riparios (más del 70\%) y los tacotales (más del 85\%) son los dos usos del suelo más importantes para la 
generación de servicios ambientales, seguidos de las pasturas mejoradas con árboles. No apareció diferencia de percepción entre los 'pobres' y 'no-pobres' (Cuadro 6). A pesar de que reconocen las bondades de los usos de la tierra forestales (bosques riparios, secundarios y tacotales) en la generación de servicios ambientales en las fincas, esta situación no se refleja en los cambios que los finqueros hacen en sus fincas para beneficiarse del PSA. Por ejemplo, la mayoría de los productores invirtió incrementando las áreas de pasturas mejoradas con alta densidad de árboles y con cercas vivas. También un número considerable (71.7\%) de los productores clasificados como 'pobres' señalaron que las cercas vivas son significativas para generar servicios ambientales. Los datos del proyecto indican que entre 2003 y 2006 el área de pasturas con árboles aumentó en 15.1\% y los productores apostaron por las cercas multiestrato. Además, las longitudes de las mismas aumentaron más del 50\% mientras que en el mismo período el área de bosque sólo se incrementó en 1.1\%. A pesar de que los productores califican el valor del bosque como decisivo para generar servicios ambientales, ellos no invierten mucha área de sus fincas en este tipo de uso debido a que el sistema del esquema del PSA implementado por el proyecto es temporal. El pago sólo fue de dos años para un grupo y de cuatro años para el otro. Por lo tanto, no quieren correr el riesgo de dejar áreas exclusivamente para conservación porque no saben si después de finalizado el proyecto tendrán pago por esas áreas. Es esta la razón que explica por qué los productores fueron muy conservadores en hacer estos cambios. Además argumentan que es necesario asegurar la sostenibilidad de los ingresos familiares provenientes de la actividad productiva de la finca (costo de oportunidad de los usos de la tierra para conservación después de finalizado el proyecto).

Cuadro 6. Valoración ambiental de los usos del suelo por parte de los productores. (Porcentaje de respuestas) 'pobres' ( $n=53)$, 'no-pobres' $(n=34)$

\begin{tabular}{|c|c|c|c|c|c|c|c|c|c|c|c|c|}
\hline $\begin{array}{l}\text { Grado de } \\
\text { importancia }\end{array}$ & $\begin{array}{r}\mathrm{Ba} \\
\text { forr }\end{array}$ & $\begin{array}{l}\text { co } \\
\text { ero }\end{array}$ & $\begin{array}{l}\text { Pas } \\
\text { natu } \\
\text { c/ár }\end{array}$ & $\begin{array}{l}\text { Iras } \\
\text { ales } \\
\text { oles }\end{array}$ & $\begin{array}{l}\text { Past } \\
\text { mejol } \\
\text { c/árl }\end{array}$ & $\begin{array}{l}\text { Iras } \\
\text { adas } \\
\text { oles }\end{array}$ & $\begin{array}{r}\text { Bos } \\
\text { secun } \\
\text { y tac }\end{array}$ & $\begin{array}{l}\text { ues } \\
\text { arios } \\
\text { ales }\end{array}$ & $\begin{array}{l}\text { Bos } \\
\text { rip }\end{array}$ & & & \\
\hline $\begin{array}{l}\text { Estrato de } \\
\text { pobreza }\end{array}$ & $\mathrm{P}$ & $\mathrm{NP}$ & $\mathrm{P}$ & $\mathrm{NP}$ & $\mathrm{P}$ & $\mathrm{NP}$ & $\mathrm{P}$ & NP & $\mathrm{P}$ & NP & $\mathrm{P}$ & NP \\
\hline $\begin{array}{l}\text { Menos } \\
\text { importante }\end{array}$ & 35.8 & 41.2 & 5.7 & 8.8 & 0.0 & 0.0 & 0.0 & 2.9 & 0.0 & 0.0 & 1.9 & 0.0 \\
\hline Importante & 32.1 & 17.6 & 47.2 & 50.0 & 26.4 & 35.3 & 13.2 & 11.8 & 18.9 & 29.4 & 26.4 & 47.1 \\
\hline $\begin{array}{l}\text { Más } \\
\text { importante }\end{array}$ & 32.1 & 41.2 & 47.2 & 41.2 & 73.6 & 64.7 & 86.8 & 85.3 & 81.1 & 70.6 & 71.7 & 52.9 \\
\hline
\end{tabular}

$\mathrm{P}=$ 'pobres', $\mathrm{NP}=$ 'no-pobres' 


\subsection{Impacto del proyecto en el nivel de ingresos de los productores}

Un alto porcentaje de los productores 'pobres' (86.8\%) y 'no-pobres' (85.3\%) mencionó que sus ingresos han incrementando durante el período en que han participado en el proyecto (Ilustración 5). Esto coincide con los datos del monitoreo socioeconómico del proyecto que indican que la producción de leche de los productores 'pobres' incrementó entre 30 y $40 \%$ el ingreso per. capita familiar entre 60 y 80\% durante el período 2003-2006 (Marín et al., 2006). Aunque el aumento en los ingresos está influenciado fuertemente por la mejoría en los precios de la leche durante este período, los aumentos en la productividad lechera y en los ingresos adicionales por el PSA parecen ser determinantes en que los productores tengan una percepción muy alta de los beneficios generados por el Proyecto en este sentido.

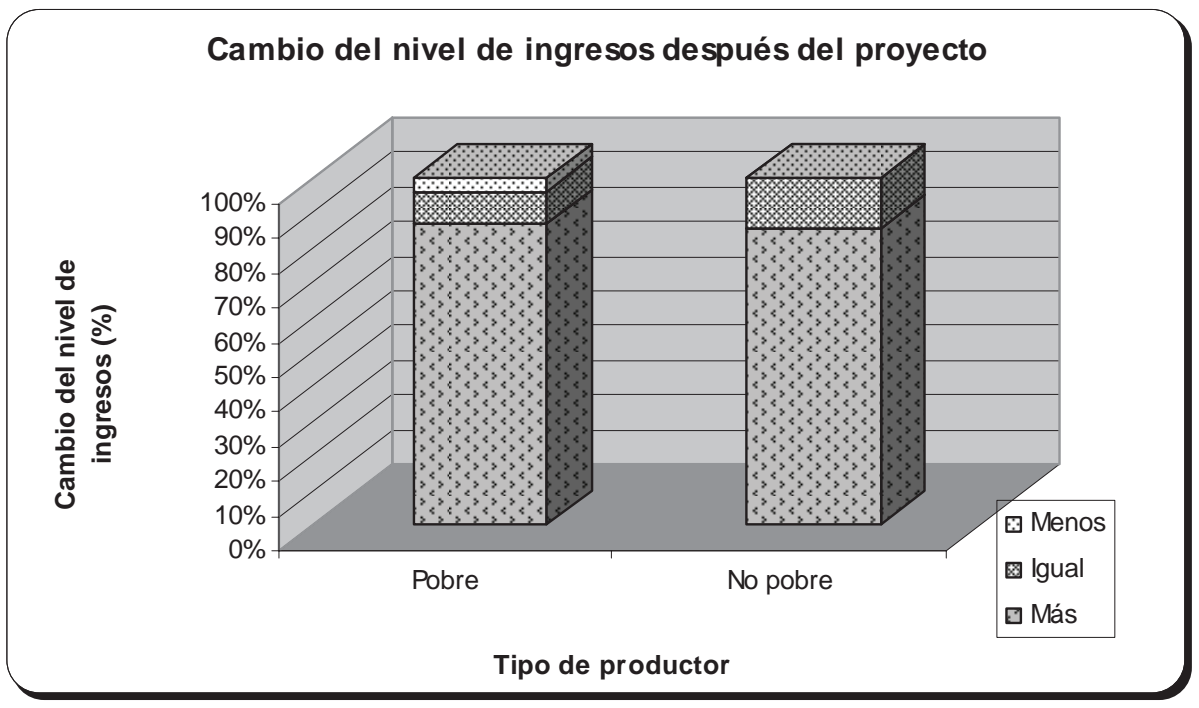

Ilustración 5. Impacto del proyecto en los ingresos de fincas 'pobres' y 'no-pobres' (porcentaje de respuestas)

\subsection{Planes de los productores}

A los productores se les preguntó también sobre sus planes de inversión en sus fincas en los próximos cinco años. Más del 51\% de los productores de ambos grupos tiene como meta incrementar la productividad de la finca; un porcentaje relativamente alto (24\%) de los productores 'pobres' y un 37\% de los 'no-pobres' quieren mejorar las condiciones ambientales y continuar generando servicios ambientales; mientras que un porcentaje menor (entre 12 y $15 \%$ ) de los dos estratos desea seguir produciendo bajo las condiciones actuales (ver la Ilustración 6).

Dadas las condiciones relativamente favorables del mercado, en general se puede anotar que a los productores del Proyecto les interesa continuar produciendo e intensificando la producción ganadera pero al mismo tiempo son más concientes de conservar los recursos 
naturales. Las ventajas que ofrecen los sistemas silvopastoriles han sido reconocidas por los productores, que en su mayoría (98\%) piensan mantener los cambios e incluso ampliarlos al resto de la finca, aun sin proyecto. No obstante, existen todavía algunas limitaciones para poder implementar más rápidamente estas tecnologías, en especial para los más 'pobres', lo que amerita el diseño de nuevos incentivos para la replicación de estos sistemas en este nicho de productores.

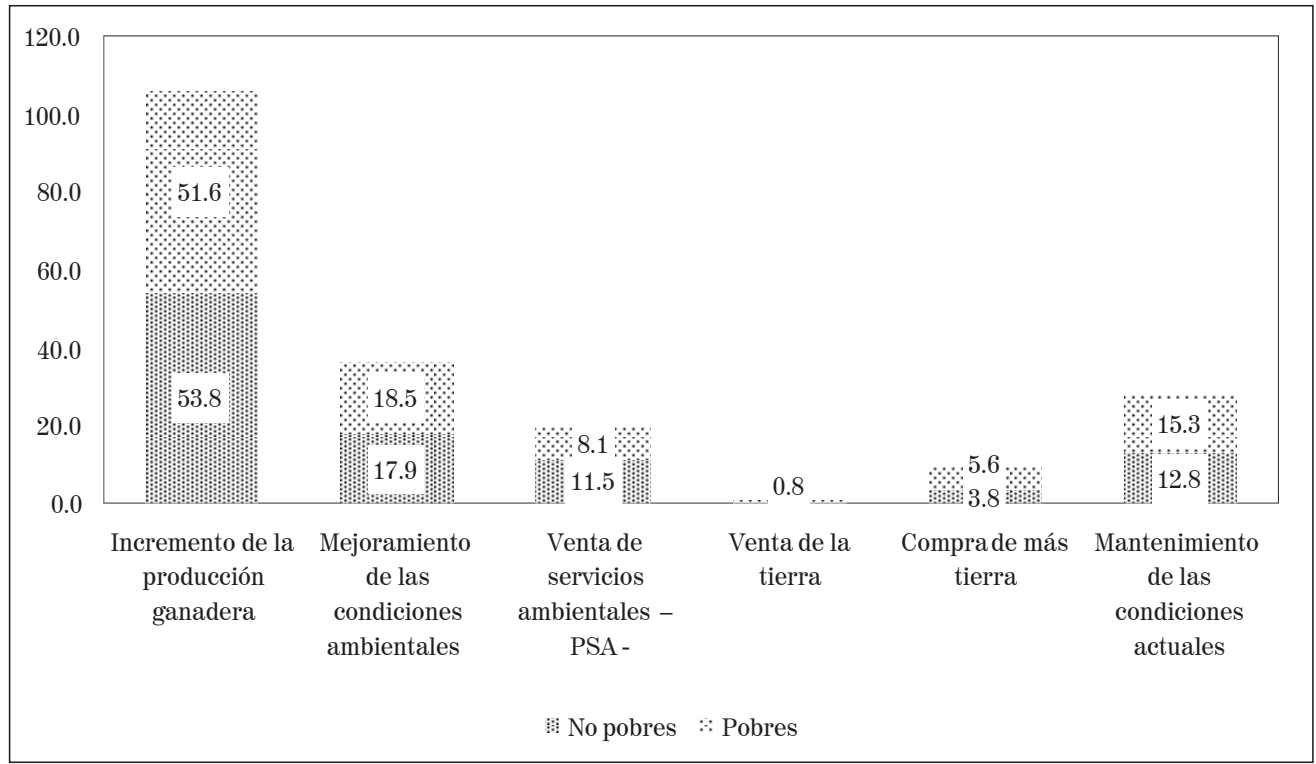

Ilustración 6. Plan de finca en los próximos cinco años con productores 'pobres' y 'nopobres' (porcentaje de respuestas)

\subsection{Incentivos que debería de fomentar el Estado para una ganadería más sostenible}

A los productores se les preguntó finalmente sobre el tipo de incentivos que debería de desarrollar el Estado para la replicación de modelos silvopastoriles y un manejo más sostenible de la producción ganadera. La Ilustración 7 muestra respuestas que estuvieron enfocadas hacia la creación de mecanismos que ofrezcan más y mejores incentivos para que los productores continúen en la actividad ganadera y puedan así romper las barreras que encuentran para la adopción de buenas prácticas de manejo silvopastoril para la generación de servicios ambientales en sus propiedades. En este sentido, el 37\% de los productores ('pobres' y 'no-pobres') considera que el Estado debería de proveer líneas de crédito verde para favorecer la implementación inicial de estos sistemas, lo que consecuentemente les generaría incrementos en la productividad, asegurándoles un mejor bienestar y mejores condiciones de vida para sus familias. Un 25\% de los finqueros considera que el Estado debe de impulsar planes de capacitación y asistencia técnica para lograr los objetivos. Un 19\% considera que el gobierno debe de seguir impulsando mecanismos de PSA para incentivar 
a otros productores a adoptar sistemas silvopastoriles con la implementación de buenas prácticas y mejores tecnologías de producción. Así los productores pueden reducir las áreas que tienen destinadas para la ganadería y liberar áreas frágiles para su reforestación, lo que permitiría una ganadería más sostenible desde el punto de vista ambiental y económicamente más rentable.

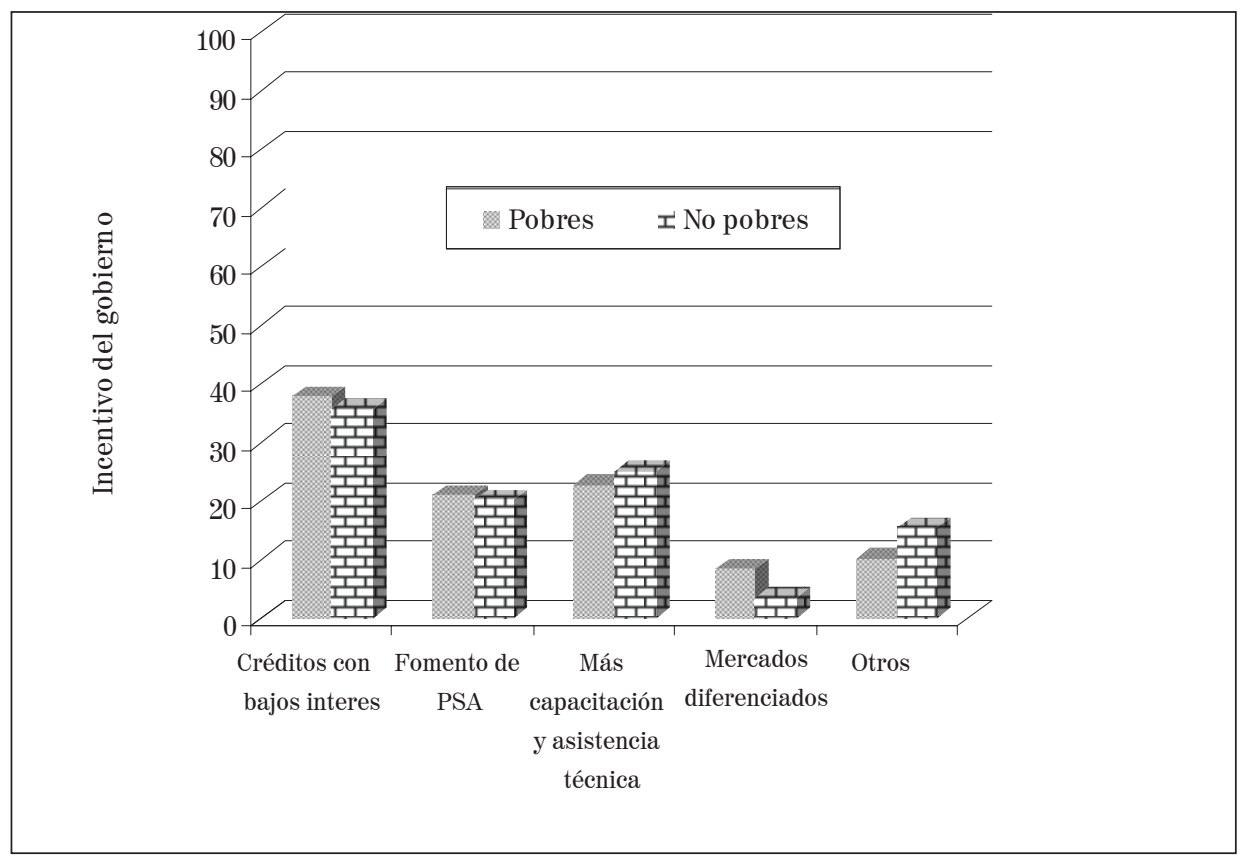

Ilustración 7. Incentivos para el fomento de una ganadería más sostenible (porcentaje de respuestas)

\section{Conclusiones y recomendaciones}

Los productores de ambos estratos de pobreza reconocen que el pago que reciben es por la generación de servicios ambientales y están satisfechos con el monto recibido. Esto significa que los productores aprovechan la oportunidad de tener ingresos adicionales - aparte de la venta de leche y ganado - para su finca y queda demostrado que los 'pobres' también pueden participar con los mismos beneficios en programas de PSA.

Además de tener impactos económicos en las fincas de productores 'pobres' y 'no-pobres', el PSA puede contribuir con el mejoramiento de los aspectos sociales de las familias, dándoles mayores recursos para invertir en educación y salud. Además, la realización de cambios de uso del suelo en las fincas puede crear más fuentes de empleo de mano de obra en el sector rural.

La ausencia de recursos financieros para la inversión en sistemas silvopastoriles representa una barrera para la generación de servicios ambientales, por lo que se debe de diseñar 
políticas de créditos blandos y rediseño o diseño de programas de capacitación y asistencia técnica permanente para el fomento y la adopción de buenas prácticas en la fincas.

Una política ganadera basada únicamente en el incremento de la producción, sin preocuparse por la preservación o incremento de los recursos naturales en las fincas, podría enfrentar problemas graves de sostenibilidad en el mediano plazo y a su vez desembocar en un aumento de la pobreza. Los PSA pueden contribuir a una producción ganadera más sostenible, diversificada y competitiva, ya que los 'pobres' rurales y sus familias pueden alcanzar un mayor bienestar.

Notas

1 En 1990, el PNUD propuso que la pobreza humana no fuera considerada únicamente como pobreza económica.

\section{Referencias bibliográficas}

BETANCOURT, H; PEZO, D; CRUZ, J. \& BEER, J. (2006). "Impacto bioeconómico de la degradación de pasturas en fincas de doble propósito en El Chal, Petén, Guatemala”. En IV Congreso Latinoamericano de Agroforestería para la producción pecuaria sostenible y III Simposio sobre sistemas silvopastoriles para la producción ganadera sostenible (Cuba). Memoria.

CASASOLA, F; IBRAHIM, M.; RAMÍREZ, E.; VILLANUEVA, C.; SEPÚLVEDA, C. \& ARAYA, J.L. (2006). "Lecciones aprendidas en el pago de servicios ambientales y los cambios en los usos de la tierra en paisajes dominados por la ganadería: Un estudio de caso en el trópico sub-húmedo de Nicaragua y Costa Rica”. En IV Congreso Latinoamericano de Agroforestería para la producción pecuaria sostenible y III Simposio sobre sistemas silvopastoriles para la producción ganadera sostenible (Cuba)No 45: 7985.

CAMACHO, M.; SEGURA, O.; REYES, V.\&MIRANDA, M. (2001). Gestión local y participación en torno al pago por servicios ambientales: Estudios de caso en Costa Rica. Proyecto PRISMA-FORD: San José.

CHAGOYA, JL. (2004). Investment Analysis of Incorporating Timber Trees in Livestock Farms in the Sub Humid Tropics of Costa Rica. Mag. Sc. Thesis, CATIE: Turrialba.

DE CAMINO, R., SEGURA, O.; ARIAS, L. \& PÉREZ, I. (1999). Forest Policy and the Evolution of Land Use: An Evaluation of Costa Rica's Forest Development and World Bank Assistance. Operations Evaluation Department: Document of the World Bank.

ESPINOZA, N.; GATICA, J. \& SMYLE, J. (1999). El Pago de Servicios Ambientales y el Desarrollo Sostenible en el Medio Rural. Serie de Publicaciones RUTA. Unidad Regional de Asistencia Técnica (RUTA). IICA: San José.

HERRADOR, D.; DIMAS, L. \& MÉNDEZ E. (2002). "Pago por servicios ambientales en El Salvador: oportunidades y riesgos para pequeños agricultores y comunidades rurales". En Proyecto Pago por Servicios Ambientales en Las Américas. Fundación FORD y Fundación PRISMA: San Salvador.

HOLMANN, F. \& ESTRADA, R.D. (1997). "Alternativas agropecuarias en la Región Pacífico Central de Costa Rica: un sistema de simulación aplicable a sistemas doble propósito”. 
En Lascano, C.E. \& Holmann, F. (Eds.) Conceptos y metodologías de Investigación en Fincas con Sistema de Producción Animal de Doble Propósito. Cali, Colombia. $\mathrm{P}$ 134-152.

IBRAHIM, M; CHACÓN, M; MORA, J; ZAMORA, S; GOBBI, J; LLANDERAL, T; HARVEY, CA; MURGUEITIO, E; CASASOLA, F; VILLANUEVA, C.\& RAMÍREZ, E. (2005). Opportunities for Carbon Secuestration and Conservation of Water Resources on Landscapes Dominated by Cattle Production in Central America. En IV Wallas conference: Integrated Management of Environmental Services in Human-Dominated Tropical Landscapes. Memoria. Turrialba, CR, CATIE. p. 27-34. (4 ${ }^{\text {th }}$ Conference in the Series).

INFOSTAT (2004). Manual del usuario. Grupo InfoStat, FCA, Universidad Nacional de Córdoba. Primera edición. Editorial Brujas: Córdoba.

LEVARD, L; MARÍN, Y. \& NAVARRO, I. (2000). Potencialidades y limitantes para el desarrollo agropecuario del municipio de Matiguás. Cuaderno de Investigación No. 11. Nitlapan-UCA: Managua.

LÓPEZ, M. (2005). El proceso de toma de decisiones en la adopción de bancos de proteína de Gliricidia sepium por productores de doble propósito en Rivas, Nicaragua. Tesis Mag. Sc., CATIE: Turrialba.

MARÍN,Y.; IBRAHIM, M.; VILLANUEVA.; C, RAMÍREZ, E. \& SEPÚLVEDA, C. (2006). "Pago por servicios ambientales en fincas ganaderas: una alternativa para la conversión de tierras y alivio de pobreza en Matiguás, Nicaragua”. En Agroforestería en las Américas. No 45: 109-116.

MUNK, H. (2004). Pobreza y degradación ambiental en las Laderas de Nicaragua. En Pobreza y deterioro Ambiental en América Latina. RIMISP: Chile.107-129 p.

ORTIZ, E. (2002). Sistema de Cobro y Pago de Servicios Ambientales en Costa Rica. Serie de Apoyo Académico No. 34.Área de Servicios Ambientales, Escuela de Ingeniería Forestal, ITCR: San José.

PAGIOLA S., AGOSTINI P, GOBBI J. DE HAAN C., IBRAHIM M., MURGUEITIO E., RAMIREZ E. ROSALES M \& RUIZ J. (2004). Paying for Biodiversity Conservation in Agricultural landscapes. Environment Department Papers, no 96: World Bank.

PAGIOLA, S; ARCENAS, A. \& PLATAIS, G. (2005). “Can Payments for Environmental Services Help Reduce Poverty? An Exploration of the Issues and the Evidence to Date From Latin America”. World Development 33:237-253.

RUIZ, GA. (2002). Fijación y almacenamiento de carbono en sistemas silvopastoriles y competitividad económica en Matiguas, Nicaragua. Tesis Mag. Sc. CATIE: Turrialba.

RODRÍGUEZ, J. (2002.) "Los servicios ambientales del bosque: el ejemplo de Costa Rica". En Revista Forestal Centroamericana 37:47-53.

SZOTT, L.; IBRAHIM, M. \& BEER, J. (2000). The Hamburger Connection Hangover. Cattle Pasture Land Degradation and Alternative Land Use in Central America. CATIE, DANIDA, GTZ: San José.

ZAPATA, A.; MURGUEITIO, E.; MEJÍA, C.; ZULUAGA, A.F. \& IBRAHIM, M. (2006). "Impacto del pago de servicios ambientales en la adopción de sistemas silvopastoriles en paisajes ganaderos de la cuenca media del río La Vieja, Colombia”. En Agroforestería en las Américas. No 45: 86-92 